\title{
THE ASYMPTOTIC DISTRIBUTION OF THE NUMBER OF ZERO FREE INTERVALS OF A STABLE PROCESS
}

\author{
BY \\ R. K. GETOOR(1)
}

1. Introduction. Let $\{X(t) ; t \geqq 0\}$ be the one-dimensional symmetric stable process of index $\alpha, 0<\alpha \leqq 2$, that is, a process with stationary independent increments whose continuous transition density $f(t, y-x)$ relative to Lebesgue measure is given by

$$
f(t, x)=(2 \pi)^{-1} \int_{-\infty}^{\infty} e^{-t|\xi|^{\alpha}} e^{i x \xi} d \xi .
$$

We assume throughout this paper that $X(0)=0$ and that the sample functions are normalized to be right continuous and have left-hand limits everywhere.

Let us also introduce the stable subordinator, $\{T(t): t \geqq 0\}$, of index $\beta$, $0<\beta<1$, that is, a process with stationary independent and positive increment whose continuous transition density, $g(t, y-x)$, is given by $g(t, x)=0$ for $x \leqq 0$ and by

$$
e^{-t s^{\beta}}=\int_{0}^{\infty} e^{-s x} g(t, x) d x
$$

for $x>0$. We assume $T(0)=0$ and that the sample functions are normalized to be right continuous and have left-hand limits everywhere. In addition almost all sample functions of $T$ are strictly monotone increasing. Finally we assume that the processes $X$ and $T$ are completely independent and are defined over th: same complete probability space, $(\Omega, \mathscr{F}, P)$. It is perhaps more reasonable to assume that $X$ and $T$ are defined over different (complete) probability spaces, but for notational convenience we prefer the above assumption.

Define

$$
\begin{aligned}
& A(\omega)=\{t: 0 \leqq t \leqq 1, X(t, \omega)=0 \text { or } X(t-, \omega)=0\} \\
& B(\omega)=\{t: 0 \leqq t \leqq 1, T(\tau, \omega)=t \text { or } T(\tau-, \omega)=t \text { for some } \tau\} .
\end{aligned}
$$

If $0<\alpha \leqq 1$ then $A(\omega)=\{0\}$ for almost all $\omega$, and it is not difficult to see that for general $\alpha, 0<\alpha \leqq 2$,

$$
A(\omega)=\{t: 0 \leqq t \leqq 1, X(t, \omega)=0\}
$$

Presented to the Society, November 6, 1961; received by the editors November 28, 1961.

(1) This research was supported, in part, by the National Science Foundation. 
for almost all $\omega$. However we will not need this fact and so we omit its proof. In view of our regularity assumptions on the sample functions of $X$ and $T$, it follows that $A(\omega)$ and $B(\omega)$ are compact subsets of $[0,1]$ for each $\omega$. Therefore the complements of $A(\omega)$ and $B(\omega)$ in [0,1] are relatively open subsets of $[0,1]$, and, as such, each can be written uniquely as the disjoint union of at most countably many (relatively) open subintervals of $[0,1]$. If $\varepsilon>0$, let $N_{\alpha}(\varepsilon)\left(N_{\beta}^{*}(\varepsilon)\right.$ ) be the number of such intervals in the complement of $A(\omega)(B(\omega))$ which exceed $\varepsilon$ in length. The following two theorems are the main results of the present paper.

THEOREM A. $N_{\alpha}(\varepsilon)$ and $N_{\beta}^{*}(\varepsilon)$ are random variables and if $\beta=1-1 / \alpha$, $1<\alpha \leqq 2$, then they have the same distribution for each fixed $\varepsilon>0$.

THEOREM B. If $0<\beta<1$, then $\lim _{\varepsilon \downarrow 0} P\left[\Gamma(1-\beta) \varepsilon^{\beta} N_{\beta}^{*}(\varepsilon) \leqq x\right]=G_{\beta}(x)$ where $G_{\beta}(x)$ is a Mittag-Leffler distribution which is uniquely determined by its moments

$$
\int_{0}^{\infty} x^{n} d G_{\beta}(x)=n ![\Gamma(1+n \beta)]^{-1}, \quad n=0,1, \ldots
$$

The definition of the distribution $G_{\beta}$ and the fact that its moments are given by (1.4) is contained in [7]. The fact that $G_{\beta}$ is uniquely determined by its moments follows from the criterion on p. 110 of [4].

An immediate consequence of Theorems A and B is the following corollary.

Corollary. If $1<\alpha \leqq 2$, then

$$
\lim _{\varepsilon \downarrow 0} P\left[\Gamma(1 / \alpha) \varepsilon^{1-1 / \alpha} N_{\alpha}(\varepsilon) \leqq x\right]=F_{\alpha}(x)=G_{1-1 / \alpha}(x) .
$$

Of course, in the case $\alpha=2$ these results are well known. Moreover the above corollary should be compared with the recent result of Kesten [5]. In [5] Kesten obtains the limiting distribution of the number, $N_{\alpha}^{\prime}(\varepsilon)$, of intervals of positivity of $X$ in $0 \leqq t \leqq 1$ for all $\alpha, 0<\alpha \leqq 2$. We would like to thank Professor Kesten for making his manuscript available to us. In particular, we owe references $[4 ; 7]$ to him.

2. The proof of Theorem $A$. Given a complete probability space $(\Omega, \mathscr{F}, P)$, a function $A$ from $\Omega$ to subsets of the real line, $R$, is said to be a random set if

(i) $A(\omega)$ is compact for almost all $\omega$,

(ii) $\{\omega: A(\omega) \subset E\} \in \mathscr{F}$ for all open subsets $E$ of $R$.

Two random sets $A$ and $B$ (not necessarily defined over the same probability space) are stochastically equivalent if for every set $E$ that is a finite union of open intervals

$$
P\{\omega: A(\omega) \subset E\}=P\{\omega: B(\omega) \subset E\}
$$


These definitions were introduced in [2]. A random set, $A$, is contained in the closed interval $[a, b]$ if $A(\omega) \subset[a, b]$ for all $\omega$. If a random set $A$ is contained in $[a, b]$ then $[a, b]-A(\omega)$ is an open subset of $[a, b]$ for almost all $\omega$, and, as such, can be written uniquely as the union of at most countably many disjoint (relatively) open subintervals of $[a, b]$. If $\varepsilon>0$, let $N_{A}(\varepsilon)$ be the number of such intervals whose length is greater than $\varepsilon$. Clearly $N_{A}(\varepsilon)$ is defined and finite for almost all $\omega$.

THEOREM 2.1. If $A$ is a random set contained in $[a, b]$, then $N_{A}(\varepsilon)$ is a random variable. If $A$ and $B$ are stochastically equivalent random sets contained in $[a, b]$ then $N_{A}(\varepsilon)$ and $N_{B}(\varepsilon)$ have the same distribution for each $\varepsilon>0$.

Proof. Let $\varepsilon>0$ be fixed, and let $k \geqq 1$ be an integer. Let $E$ denote a finite disjoint union of exactly $k$ closed intervals $I_{1}, \cdots, I_{k}$ each of which has rational end points, is contained in $[a, b]$, and has length greater than $\varepsilon$. Of course, if $k$ is too large there will be no such $E$ 's. Let $E_{1}, E_{2}, \cdots$ be an enumeration of all such $E$ 's; then ( $\varnothing$ denotes the empty set)

$$
\left\{\omega: N_{A}(\varepsilon) \geqq k\right\}=\bigcup_{n=1}^{\infty} \Delta_{n},
$$

where

$$
\Delta_{n}=\left\{\omega: E_{n} \cap A(\omega)=\emptyset\right\}=\left\{\omega: A(\omega) \subset E_{n}^{c}\right\} .
$$

Here $E_{n}^{c}$ is the complement of $E_{n}$ in $[a, b]$ and hence is a finite union of (relatively) open subintervals of $[a, b]$. Clearly this implies that $N_{A}(\varepsilon)$ is a random variable. Moreover

$$
P\left\{\omega: N_{A}(\varepsilon) \geqq k\right\}=\lim _{n \rightarrow \infty} P\left(\bigcup_{i=1}^{n} \Delta_{i}\right),
$$

and for fixed $n$ the inclusion-exclusion formula implies that

$$
P\left(\bigcup_{i=1}^{n} \Delta_{i}\right)=\Sigma P\left(\Delta_{i}\right)-\Sigma P\left(\Delta_{i} \cap \Delta_{j}\right)+\cdots .
$$

Looking at a typical intersection we see that

$$
P\left(\Delta_{i} \cap \ldots \cap \Delta_{j}\right)=P\left\{\omega: A(\omega) \subset E_{i}^{c} \cap \ldots \cap E_{j}^{c}\right\} .
$$

Since $E_{i}^{c} \cap \ldots \cap E_{j}^{c}$ is a finite union of open intervals (not necessarily disjoint), it follows that if $A$ and $B$ are stochastically equivalent the left side of (2.4) is unchanged if $A$ is replaced by $B$. Thus $N_{A}(\varepsilon)$ and $N_{B}(\varepsilon)$ have the same distribution.

Let $a$ be a real number satisfying $0 \leqq a<1$, and define

$$
\begin{aligned}
& A_{a}(\omega)=\{t: a \leqq t \leqq 1, X(t, \omega)=0 \text { or } X(t-, \omega)=0\}, \\
& B_{a}(\omega)=\{t: a \leqq t \leqq 1, T(\tau, \omega)=t \text { or } T(\tau-, \omega)=t \text { for some } \tau\},
\end{aligned}
$$


where $X$ and $T$ are the processes defined in $\S 1$. We assume from now on that the index, $\alpha$, of $X$ satisfies $1<\alpha \leqq 2$. In particular, $A_{0}$ and $B_{0}$ are the sets $A$ and $B$ defined in (1.3). It was shown in [2, Proof of Theorem A] that $A_{a}$ and $B_{a}$ are stochastically equivalent random sets for each $a>0$ provided $\beta=1-1 / \alpha$. Thus by Theorem 2.1 we see that $N_{\alpha}(a, \varepsilon)=N_{A_{a}}(\varepsilon)$ and $N_{\beta}^{*}(a, \varepsilon)=N_{B_{a}}(\varepsilon)$ have the same distribution for each fixed $a>0$ and $\varepsilon>0$ provided $\beta=1-1 / \alpha$. Since for almost all $\omega$ the sets $A(\omega)$ and $B(\omega)$ contain points arbitrarily close to 0 (this is an immediate consequence of Lemma 3.1 of [2]), it follows that $N_{\alpha}(a, \varepsilon) \rightarrow N_{\alpha}(\varepsilon)$ and $N_{\beta}^{*}(a, \varepsilon) \rightarrow N_{\beta}^{*}(\varepsilon)$ as $a \rightarrow 0$ for almost all $\omega$. In fact for $a$ sufficiently small (depending on $\varepsilon$ and $\omega$ ) we have $N_{\alpha}(a, \varepsilon, \omega)=N_{\alpha}(\varepsilon, \omega)$ and similarly for $N^{*}$. Thus $N_{\alpha}(\varepsilon)$ and $N_{\beta}^{*}(\varepsilon)$ have the same distribution if $\beta=1-1 / \alpha$, and Theorem $\mathrm{A}$ is proved.

3. First passage times. In this section we give a preliminary calculation that will be needed in the proof of Theorem B. Let $T=\{T(t) ; t \geqq 0\}$ be the stable subordinator of index $\beta, 0<\beta<1$, and we assume in this section that $T(0)=0$. Let us recall the Ito representation of $T$ (see [3] or [6, \$37]). In the present case $(T(t)$ strictly increasing) this is especially simple. For fixed $\omega$ let $p(d t, d x, \omega)$ be the measure on $[0, \infty) \times(0, \infty)$ defined by the relationship that

$$
p\left(\left(t_{1}, t_{2}\right],\left(x_{1}, x_{2}\right], \omega\right)
$$

is the number of points $\tau, t_{1}<\tau \leqq t_{2}$ such that $[T(\tau, \omega)-T(\tau-, \omega)] \in\left(x_{1}, x_{2}\right]$. Here $0 \leqq t_{1}<t_{2}$ and $0<x_{1}<x_{2}$. The measure, $p$, is called the Poisson measure of $T$. The random variable $p(d t, d x)$ has a Poisson distribution with expected value $d t v(d x)$ where $v(d x)=\beta\left[\Gamma(1-\beta) x^{1+\beta}\right]^{-1} d x$ is the Lévy measure of $T$. See $[1, \S 6]$. Moreover

$$
T(t, \omega)=\int_{0+}^{\infty} x p([0, t], d x, \omega),
$$

where in this case the integral is just the countable sum of the jumps of $T(\tau, \omega)$ on the interval $0 \leqq \tau \leqq t$. Finally the random variable $p([t, s), d x), s>t$, is independent of $\mathscr{B}_{t-}$, the $\sigma$-algebra generated by $\{T(\tau): \tau<t\}$, and if $A_{1}, \ldots, A_{n}$ are disjoint Borel subsets of $\{(t, x): t \geqq 0, x>0\}$ which are at a positive distance from the $t$-axis, then $\int_{A_{1}} p(d t, d x), \ldots, \int_{A_{n}} p(d t, d x)$ are independent random variables.

If $u>0$ define

$$
S(u, \omega)=\inf \{t: T(t, \omega) \geqq u\} .
$$

Since $T(t)$ is strictly increasing, $S(u)$ is continuous and nondecreasing. It is the first passage time of $T$ past $u$. We now state the main result of this section.

THEOREM 3.1. For each $u>0$ and integer $k \geqq 0$ we have

$$
E\left(S(u)^{k}\right)=k ![\Gamma(1+\beta k)]^{-1} u^{\beta k} .
$$


Proof. For $\lambda>0$ and $s>0$, define

$$
H_{k}(\lambda, s)=E \int_{0}^{\infty} e^{-\lambda S(u)^{k}} e^{-s u} d u .
$$

Now $T(t, \omega)$ is a sum of jumps and so if we let $t_{n}$ be the places where $T(t)$ jumps and $I_{n}=\left[T\left(t_{n}-\right), T\left(t_{n}\right)\right)$ then $\bigcup I_{n}=[0, \infty)$ since $T(0)=0$ and $T(t) \rightarrow \infty$ as $t \rightarrow \infty$. Of course, the $t_{n}$ depend on $\omega$. For notational convenience let us write $T^{*}(t)$ for $T(t-)$. Thus

$$
\begin{aligned}
\int_{0}^{\infty} e^{-\lambda S(u)^{k}} e^{-s u} d u & =\frac{1}{s} \sum_{n} e^{-\lambda t_{n}^{k}}\left[e^{-s T^{*}\left(t_{n}\right)}-e^{-s T\left(t_{n}\right)}\right] \\
& =\frac{1}{s} \sum_{n} e^{-\lambda t_{n}^{k}} e^{-s T^{*}\left(t_{n}\right)}\left[1-e^{-s\left[T\left(t_{n}\right)-T^{*}\left(t_{n}\right)\right]}\right] \\
& =\frac{1}{s} \int_{0}^{\infty} \int_{0}^{\infty} e^{-\lambda t^{k}} e^{-s T^{*}(t)}\left[1-e^{-s x}\right] p(d t, d x) .
\end{aligned}
$$

But $T^{*}(t)$ and $p(d t, d x)$ are independent and

$$
E\left[\exp \left(-s T^{*}(t)\right)\right]=E[\exp (-s T(t))]=e^{-t s^{\beta}}
$$

for fixed $t$. Therefore (these manipulations are easily justified by first approximating the integral by a sum and then passing to the limit)

$$
\begin{aligned}
H_{k}(\lambda, s) & =\frac{1}{s} \int_{0}^{\infty} \int_{0}^{\infty} e^{-\lambda t^{k}} e^{-t s^{\beta}}\left(1-e^{-s x}\right) \frac{\beta d t d x}{\Gamma(1-\beta) x^{1+\beta}} \\
& =s^{\beta-1} \int_{0}^{\infty} e^{-\lambda t^{k}} e^{-t s^{\beta}} d t .
\end{aligned}
$$

Differentiating with respect to $\lambda$ and letting $\lambda \rightarrow 0$ we obtain (the interchange of limit procedures is easily justified)

$$
\begin{aligned}
\int_{0}^{\infty} E\left[S(u)^{k}\right] e^{-s u} d u & =s^{\beta-1} \int_{0}^{\infty} t^{k} e^{-t s^{\beta}} d t \\
& =k ! s^{-1-\beta k} .
\end{aligned}
$$

Inverting this Laplace transform we obtain Theorem 3.1.

It is an easy consequence of this theorem that the distribution function of $S(u)$ is $G_{\beta}\left(u^{-\beta} x\right)$ where $G_{\beta}$ is defined in (1.4).

4. Proof of Theorem B. Let $M(\varepsilon)$ be the number of jumps of $T(t)$ in the interval $0 \leqq t \leqq S(1)$ of length greater than $\varepsilon$. Thus if $Q(d t, \varepsilon)=p(d t,(\varepsilon, \infty))$ where $p$ is the Poisson measure for $T(t)$, then $M(\varepsilon)=Q\left(\left[0, S_{1}\right], \varepsilon\right)$. Of course, $S_{1}=S(1)$ 
is defined in (3.2). Clearly $0 \leqq M(\varepsilon)-N_{\beta}^{*}(\varepsilon) \leqq 1$ for almost all $\omega$. We will prove that

$$
E\left\{\left[\Gamma(1-\beta) \varepsilon^{\beta} M(\varepsilon)\right]^{k}\right\} \rightarrow k ![\Gamma(1+\beta k)]^{-1}
$$

as $\varepsilon \rightarrow 0$ for each integer $k \geqq 0$. From this the corresponding relation with $M(\varepsilon)$ replaced by $N_{\beta}^{*}(\varepsilon)$ follows easily, and then Theorem B is a consequence of the moment convergence theorem for distributions $[4, \mathrm{p}$. 115]. Thus the proof of Theorem B reduces to the proof of (4.1). Note that $M(\varepsilon) \leqq\left[\varepsilon^{-1}\right]+2$, where $[\cdot]$ is the greatest integer function, and so all moments of $M(\varepsilon)$ exist.

It will be convenient to consider the subordinator $T(t)$ starting not only at 0 but also at any $x \geqq 0$. We will write $P_{x}$ and $E_{x}$ for probabilities and expectations when $T(0)=x$, and, as is usual in the general theory of Markov processes, $E_{T(t)}\{\}$ stands for the evaluation of the function $E_{x}\{\}$ at the point $x=T(t)$. Let $G(t, x, A)$ be the transition probability function for $T(t)$, and $U(x, A)$ $=\int_{0}^{\infty} G(t, x, A) d t$ be the potential kernel. Since $U(x, A)$ is just the expected amount of time $T(t)$ spends in $A$ when $T(0)=x$, we have

$$
U(x,[0, y])=E_{x}(S(y))=E_{0}(S(y-x))
$$

provided $y>x$. Thus Theorem 3.1 implies

$$
U(x, d y)= \begin{cases}\beta[\Gamma(1+\beta)]^{-1}(y-x)^{\beta-1} d y & \text { if } y>x \\ 0 & \text { if } y \leqq x .\end{cases}
$$

We begin our calculations with several lemmas. Recall that $T^{*}(t)=T(t-)$ and $\mathscr{B}_{t}$ is the $\sigma$-algebra generated by $T(\tau)$ for $\tau \leqq t$.

LEMMA 1. Let $g$ be a bounded Baire function with compact support; then

$$
\int_{0}^{\infty} E_{x}\left[g\left(T_{t}^{*}\right)\right] d t=\int_{0}^{\infty} E_{x}\left[g\left(T_{t}\right)\right] d t=\frac{\beta}{\Gamma(1+\beta)} \int_{x}^{\infty}(y-x)^{\beta-1} g(y) d y .
$$

Proof. Since $T^{*}(t)=T(t)$ for almost all $\omega$ for each fixed $t$, we have using (4.2)

$$
\begin{aligned}
\int_{0}^{\infty} E_{x}\left[g\left(T_{t}^{*}\right)\right] d t & =\int_{0}^{\infty} E_{x}\left[g\left(T_{t}\right)\right] d t \\
& =\int_{0}^{\infty} d t \int G(t, x, d y) g(y)=\frac{\beta}{\Gamma(1+\beta)} \int_{x}^{\infty}(y-x)^{\beta-1} g(y) d y .
\end{aligned}
$$

It will be convenient to introduce the following conventions. Let $0 \leqq a<b<\infty$; then $\int_{a}^{b}$ and $\int_{a}^{\infty}$ are the integrals over the sets $(a, b]$ and $(a, \infty)$, respectively. Also let

$$
h(u)= \begin{cases}1 & \text { if } u \leqq 1 \\ 0 & \text { if } u>1\end{cases}
$$


LEMMA 2. Let $\phi$ be a bounded non-negative continuous function; then

$$
E_{x} \int_{0}^{\infty} h\left(T_{u}^{*}\right) \phi\left(T_{u}^{*}\right) Q(d u, \varepsilon)=\lambda E_{x} \int_{0}^{\infty} h\left(T_{u}^{*}\right) \phi\left(T_{u}^{*}\right) d u,
$$

where $\lambda=\left[\varepsilon^{\beta} \Gamma(1-\beta)\right]^{-1}$.

Proof. Since $T_{u}^{*} \leqq 1$ if and only if $u \leqq S_{1}$, we see that $\int_{0}^{\infty} h\left(T_{u}^{*}\right) \phi\left(T_{u}^{*}\right) Q(d u, \varepsilon)$ $\leqq M(\varepsilon) \sup \phi \leqq\left(\left[\varepsilon^{-1}\right]+2\right) \sup \phi$, and $\int_{0}^{\infty} h\left(T_{u}^{*}\right) \phi\left(T_{u}^{*}\right) d u \leqq S_{1}$ sup $\phi$. Thus both integrals exist for almost all $\omega$ and have finite expectations. The result now follows by a straightforward approximation argument making use of the easily verified facts that (i) $T_{u}^{*}$ and $Q([u, v), \varepsilon), v>u$, are independent, (ii) $h\left(T_{u}^{*}\right) \phi\left(T_{u}^{*}\right)$ is left continuous in $u$, and (iii) $E_{x} Q(d u, \varepsilon)=E_{x} p(d u,(\varepsilon, \infty))=\lambda d u$.

LEMMA 3. For each $\varepsilon>0$ let $Y^{\varepsilon}=\left\{Y_{u}^{\varepsilon} ; u \geqq 0\right\}$ be a stochastic process defined on the sample space of $\{T(t) ; t \geqq 0\}$ with right continuous sample functions. Let $H_{x}(u, \omega)$ be a fixed version of $E_{x}\left\{Y_{u}^{\varepsilon} \mid \mathscr{B}_{u}\right\}$ defined for $u \geqq 0$ and all $\omega$ which is assumed to satisfy $H_{x}(u, \omega)=g\left(T_{u}\right)+O(\gamma(\varepsilon))$ where $g$ is a bounded continuous function and the $O$-term is uniform in $u, \omega$, and $x$. Finally assume that $0 \leqq Y_{u}^{\varepsilon}(\omega) \leqq M_{\varepsilon}<\infty$ for all $u, \omega$. Under these conditions

$$
E_{x} \int_{0}^{\infty} h\left(T_{u}^{*}\right) Y_{u}^{\varepsilon} Q(d u, \varepsilon)=E_{x} \int_{0}^{\infty} h\left(T_{u}^{*}\right) g\left(T_{u}\right) Q(d u, \varepsilon)+O\left(\varepsilon^{-\beta} \gamma(\varepsilon)\right)
$$

where the 0 -term is uniform in $x$.

Proof. Let $0<b<\infty$ and let ${ }_{N} t_{j}=j b / N, j=0,1, \ldots, N$, for each integer $N>0$. Let $Z(u)=h\left(T_{u}^{*}\right)$, then since $Z(u)$ is left continuous and $Y(u)=Y_{u}^{\varepsilon}$ is right continuous we have for each fixed $\varepsilon>0$ that

$$
\begin{aligned}
J(b) & =E_{x} \int_{0}^{b} Z(u) Y_{u}^{\varepsilon} Q(d u, \varepsilon) \\
& =\lim _{N \rightarrow \infty} \sum_{j=0}^{N-1} E_{x}\left\{Z\left({ }_{N} t_{j}\right) Y\left({ }_{N} t_{j+1}\right) Q\left(\Delta_{N} t_{j}, \varepsilon\right)\right\}
\end{aligned}
$$

where $\Delta_{N} t_{j}=\left({ }_{N} t_{j}, t_{j+1}\right]$. But $Z\left({ }_{N} t_{j}\right)$ and $Q\left(\Delta_{N} t_{j}, \varepsilon\right)$ are $\mathscr{B}\left({ }_{N} t_{j+1}\right)$ measurable and so using our fixed version of $E_{x}\left\{Y_{u}^{\ell} \mid \mathscr{B}_{u}\right\}$ we have

$$
\begin{aligned}
J(b) & =\lim _{N \rightarrow \infty} E_{x} \sum_{j=\infty}^{N-1} Z\left({ }_{N} t_{j}\right)\left[g\left(T\left({ }_{N} t_{j+1}\right)\right)+O(\gamma(\varepsilon))\right] Q\left(\Delta_{N} t_{j}, \varepsilon\right) \\
& =E_{x} \int_{0}^{b} h\left(T_{u}^{*}\right) g\left(T_{u}\right) Q(d u, \varepsilon)+O(\gamma(\varepsilon)) E_{x} \int_{0}^{b} h\left(T_{u}^{*}\right) Q(d u, \varepsilon) .
\end{aligned}
$$

Using Lemmas 1 and 2 we see that

$$
E_{x} \int_{0}^{\infty} h\left(T_{u}^{*}\right) Q(d u, \varepsilon)=\lambda E_{x} \int_{0}^{\infty} h\left(T_{u}^{*}\right) d u=\lambda[\Gamma(1+\beta)]^{-1}(1-x)^{\beta} h(x)=O\left(\varepsilon^{-\beta}\right)
$$


uniformly in $x$. Thus letting $b \rightarrow \infty$ in the above expression for $J(b)$ we obtain Lemma 3.

We are now ready to begin the proof of (4.1). Since $t \leqq S_{1}$ if and only if $T^{*}(t) \leqq 1$ we may write ( $Q$ assigns no mass to 0 , so $\int_{0}^{\infty}$ - equals $\int_{0}^{\infty}$ )

$$
M(\varepsilon)=\int_{0}^{\infty} h\left(T^{*}(u)\right) Q(d u, \varepsilon) .
$$

Using Lemma 2 with $\phi \equiv 1$ and then Lemma 1 we obtain

$$
E_{y}(M(\varepsilon))=E_{y}\left\{p\left(\left[0, S_{1}\right],(\varepsilon, \infty)\right)\right\}=\frac{\lambda}{\Gamma(1+\beta)}(1-y)^{\beta} h(y) .
$$

Setting $y=0$ and recalling that $\lambda=\left[\varepsilon^{\beta} \Gamma(1-\beta)\right]^{-1}$ we have established (4.1) when $k=1$.

In order to fix the ideas let us consider the case $k=2$ before proceeding to the general case. Now

$$
\begin{aligned}
E_{0}\left(M(\varepsilon)^{2}\right)= & 2 E_{0} \int_{0}^{\infty} h\left(T_{t}^{*}\right) Q(d t, \varepsilon) \int_{t}^{\infty} h\left(T_{u}^{*}\right) Q(d u, \varepsilon) \\
& +E_{0} \iint_{0 \leqq t=u} h\left(T_{t}^{*}\right) h\left(T_{u}^{*}\right) Q(d t, \varepsilon) Q(d u, \varepsilon) .
\end{aligned}
$$

But for each $\omega, Q$ is a purely discrete measure assigning mass one to each point of a countable set (depending on $\omega$ ) and $h^{2}=h$. Therefore the second term in (4.5) reduces to $E_{0}(M(\varepsilon))=O\left(\varepsilon^{-\beta}\right)$ by (4.4). Let us consider the first term.

Define $Y_{t}^{\varepsilon}=\int_{t}^{\infty} h\left(T_{u}^{*}\right) Q(d u, \varepsilon)$; then $Y_{t}^{\varepsilon}$ is right continuous since the integral is over the open interval $(t, \infty)$, and is bounded by $M(\varepsilon) \leqq\left[\varepsilon^{-1}\right]+2$. Since $T(t)$ is a Markov process we note that

$$
E_{x}\left\{Y_{t}^{\varepsilon} \mid \mathscr{B}_{t}\right\}=E_{T(t)} \int_{0}^{\infty} h\left(T_{u}^{*}\right) Q(d u, \varepsilon)
$$

for almost all $\omega\left(P_{x}\right)$. Thus using Lemmas 2 and 1 we can take as our fixed version of $E_{x}\left\{Y_{t}^{e} \mid \mathscr{B}_{t}\right\}$ the expression $\lambda q_{1}\left(T_{t}\right)$ where it is understood that $T(0)=x$, and $q_{1}(x)=[\Gamma(1+\beta)]^{-1}(1-x)^{\beta} h(x)$. Therefore the hypotheses of Lemma 3 are satisfied with $\gamma(\varepsilon)=0$. Applying Lemma 3 with $x=0$ the first term in (4.5) becomes

$$
\begin{aligned}
& 2 \lambda E_{0} \int_{0}^{\infty} h\left(T_{t}^{*}\right) q_{1}\left(T_{t}\right) Q(d t, \varepsilon) \\
& \quad=2 \lambda E_{0} \int_{0}^{\infty} h\left(T^{*}\right) q_{1}\left(T_{t}^{*}\right) Q(d t, \varepsilon)-2 \lambda E_{0} \int_{0}^{\infty} h\left(T_{t}^{*}\right)\left[q_{1}\left(T_{t}^{*}\right)-q_{1}\left(T_{t}\right)\right] Q(d t, \varepsilon) .
\end{aligned}
$$


Call the first term above $J_{1}$ and the second $J_{2}$. Using Lemmas 2 and 1 , and then the definition of $q_{1}$, we find

$$
\begin{aligned}
J_{1} & =2 \lambda^{2} \beta[\Gamma(1+\beta)]^{-1} \int_{0}^{1} y^{\beta-1} q_{1}(y) d y \\
& =2 \lambda^{2}[\Gamma(1+2 \beta)]^{-1} .
\end{aligned}
$$

Concerning $J_{2}$, we note that $q_{1}$ is bounded by $c=[\Gamma(1+\beta)]^{-1}$ and satisfies $0 \leqq q_{1}(x)-q_{1}(x+y) \leqq c y^{\beta}$ for all $x \geqq 0$ and $y \geqq 0$. Moreover there can be at most one jump of $T(t)$ of magnitude greater than one in the interval $0 \leqq t \leqq S_{1}$ and so

$$
\left|J_{2}\right| \leqq 4 c \lambda+2 \lambda c E_{0} \int_{0}^{s_{1}}\left(T_{t}-T_{t}^{*}\right)^{\beta} p(d t,(\varepsilon, 1]) .
$$

But this last integral is just $E_{0} \int_{\varepsilon}^{1} x^{\beta} p\left(\left[0, S_{1}\right], d x\right)$, and (4.4) implies that

$$
E_{0}\left\{p\left(\left[0, S_{1}\right], d x\right)\right\}=\beta[\Gamma(1+\beta) \Gamma(1-\beta)]^{-1} x^{-\beta-1} d x .
$$

Thus using a simple approximation procedure we have that

$$
\left|J_{2}\right| \leqq c_{1} \lambda+c_{2} \lambda \int_{\varepsilon}^{1} x^{\beta} x^{-1-\beta} d x=O\left(\varepsilon^{-\beta}|\log \varepsilon|\right) .
$$

Combining these estimates we finally obtain $E_{0}\left(M(\varepsilon)^{2}\right)=2 \lambda^{2}[\Gamma(1+2 \beta)]^{-1}$ $+O\left(\varepsilon^{-\beta}|\log \varepsilon|\right)$, and this implies (4.1) in the case $k=2$.

In order to attack the general case it will be necessary to introduce some notation. As above let $q_{1}(x)=E_{x} \int_{0}^{\infty} h\left(T_{t}^{*}\right) d t=[\Gamma(1+\beta)]^{-1}(1-x)^{\beta} h(x)$, and define

$$
q_{n}(x)=E_{x} \int_{0}^{\infty} h\left(T_{t}^{*}\right) q_{n-1}\left(T_{t}^{*}\right) d t
$$

for $n \geqq 2$. Let us show by induction that

$$
q_{n}(x)=[\Gamma(1+n \beta)]^{-1}(1-x)^{n \beta} h(x) .
$$

This is true when $n=1$, assuming it for $n-1$ and using Lemma 1 we find

$$
\begin{aligned}
q_{n}(x) & =\frac{\beta}{\Gamma(1+\beta)} \int_{x}^{\infty}(y-x)^{\beta-1} q_{n-1}(y) d y \\
& =[\Gamma(1+n \beta)]^{-1}(1-x)^{n \beta} h(x),
\end{aligned}
$$

and so (4.8) is established.

Secondly letting $q_{0}(x) \equiv 1$ we define

$$
L_{n}(t, \omega)=\lambda^{n-1} E_{T(t)} \int_{0}^{\infty} h\left(T_{u}^{*}\right) q_{n-1}\left(T_{u}\right) Q(d u, \varepsilon)
$$

for $n \geqq 1$. Of course, $L_{n}$ depends on $\varepsilon$. Next we show that 


$$
L_{n}(t)=\lambda^{n} q_{n}\left(T_{t}\right)+O\left(\varepsilon^{-(n-1) \beta}|\log \varepsilon|\right)
$$

for $n \geqq 1$, where the $O$-term is uniform in $t$ and $\omega$. If $n=1$, applying Lemma 2 and the definition of $q_{1}$, we have

$$
L_{1}(t, \omega)=E_{T(t)} \int_{0}^{\infty} h\left(T_{u}^{*}\right) Q(d u, \varepsilon)=\lambda q_{1}\left(T_{t}\right)
$$

which certainly implies (4.10) when $n=1$. For $n \geqq 2$ we can write

$$
\begin{aligned}
L_{n}(t)= & \lambda^{n-1} E_{T(t)} \int_{0}^{\infty} h\left(T_{u}^{*}\right) q_{n-1}\left(T_{u}^{*}\right) Q(d u, \varepsilon) \\
& -\lambda^{n-1} E_{T(t)} \int_{0}^{\infty} h\left(T_{u}\right)\left[q_{n-1}\left(T_{u}^{*}\right)-q_{n-1}\left(T_{u}\right)\right] Q(d u, \varepsilon) \\
= & J_{1}-J_{2} .
\end{aligned}
$$

From Lemma 2 and (4.7) we see that $J_{1}=\lambda^{n} q_{n}\left(T_{t}\right)$. Concerning $J_{2}$, we note $q_{n-1}$ is bounded, say by $M$, and that $0 \leqq q_{n-1}(x)-q_{n-1}(x+y) \leqq c y^{\beta}$ if $n \geqq 2$, since $0<\beta<1$. Thus exactly as in the case $k=2$ we obtain

$$
J_{2} \leqq 2 \lambda^{n-1} M+\lambda^{n-1} E_{T(t)} \int_{\varepsilon}^{1} x^{\beta} p\left(\left[0, S_{1}\right], d x\right)
$$

But from (4.4) with $y=T(t)$ we see that

$$
E_{T(t)} p\left(\left[0, S_{1}\right], d x\right)=c_{1}\left(1-T_{t}\right)^{\beta} h\left(T_{t}\right) x^{-1-\beta} d x,
$$

and so $J_{2}=O\left(\varepsilon^{-(n-1) \beta}|\log \varepsilon|\right)$ uniformly in $t$ and $\omega$ since $\left(1-T_{t}\right)^{\beta} h\left(T_{t}\right) \leqq 1$. Combining these results we obtain (4.10).

Finally we will show by induction that

$$
L_{n}(t)=E_{T(t)} \int_{0}^{\infty} h\left(T_{t_{i}}^{*}\right) Q\left(d t_{1}, \varepsilon\right) \int_{t_{1}}^{\infty} \cdots \int_{t_{n-1}}^{\infty} h\left(T_{t}^{*}\right) Q\left(d t_{n}, \varepsilon\right)+O\left(\varepsilon^{-(n-1) \beta}|\log \varepsilon|\right)
$$

provided $n \geqq 1$, where again the $O$-term is uniform in $t$ and $\omega$. Denote the first term on the right-hand side on (4.11) by $J_{n}(t)$. If $n=1,(4.11)$ is immediate from the definition of $L_{1}$. Assume (4.11) for $n-1$. Define

$$
Y^{\varepsilon}\left(t_{1}\right)=\int_{t_{1}}^{\infty} h\left(T_{t_{2}}^{*}\right) Q\left(d t_{2}, \varepsilon\right) \int_{t_{2}}^{\infty} \cdots \int_{t_{n-1}}^{\infty} h\left(T_{t_{n}}^{*}\right) Q\left(d t_{n}, \varepsilon\right)
$$

Clearly $Y^{\varepsilon}\left(t_{1}\right)$ is right continuous since the integral is over $\left(t_{1}, \infty\right)$, and $0 \leqq Y^{\varepsilon}\left(t_{1}\right) \leqq\left(\left[\varepsilon^{-1}\right]+2\right)^{n-1}$. Also since $T(t)$ is a Markov process it is easy to see that for each $y$ a version of $E_{y}\left(Y^{*}\left(t_{1}\right) \mid \mathscr{B}_{t_{1}}\right)$ is given by (T starts from $y$ )

$$
\begin{aligned}
E_{T\left(t_{1}\right)} \int_{0}^{\infty} h\left(T_{t_{2}}^{*}\right) Q\left(d t_{2}, \varepsilon\right) & \int_{t_{2}}^{\infty} \cdots \int_{t_{n-1}}^{\infty} h\left(T_{t}^{*}\right) Q\left(d t_{n}, \varepsilon\right) \\
& =L_{n-1}\left(t_{1}\right)+O\left(\varepsilon^{-(n-2) \beta}|\log \varepsilon|\right) \\
& =\lambda^{n-1} q_{n-1}\left(T_{t_{1}}\right)+O\left(\varepsilon^{-(n-2) \beta}|\log \varepsilon|\right)
\end{aligned}
$$


where we have used the induction hypothesis and (4.10). Applying Lemma 3 whose hypotheses are satisfied we find that

$$
\begin{aligned}
J_{n}(t) & =E_{T(t)} \int_{0}^{\infty} h\left(T_{t_{1}}^{*}\right) Y^{\varepsilon}\left(t_{1}\right) Q\left(d t_{1}, \varepsilon\right) \\
& =\lambda^{n-1} E_{T(t)} \int_{0}^{\infty} h\left(T_{t_{1}}^{*}\right) q_{n-1}\left(T_{t_{1}}\right) Q\left(d t_{1}, \varepsilon\right)+O\left(\varepsilon^{-(n-1) \beta}|\log \varepsilon|\right)
\end{aligned}
$$

which is (4.11) in view of the definition (4.9) of $L_{n}(t)$.

We are now ready to prove (4.1) for general values of $k \geqq 2$. Assume (4.1) for $0,1, \ldots, k-1 ;$ then

$$
\begin{aligned}
E_{0}\left(M(\varepsilon)^{k}\right)= & E_{0}\left(\int_{0}^{\infty} h\left(T_{u}^{*}\right) Q(d u, \varepsilon)\right)^{k} \\
= & k ! E_{0} \int_{0}^{\infty} h\left(T_{t_{1}}^{*}\right) Q\left(d t_{1}, \varepsilon\right) \quad \int_{t_{1}}^{\infty} \cdots \int_{t_{k-1}}^{\infty} h\left(T_{t_{k}}^{*}\right) Q\left(d t_{k}, \varepsilon\right) \\
& + \text { error term. }
\end{aligned}
$$

Now the error term consists of a finite sum of integrals over lower dimensional hyperplanes obtained by identifying a subset of the variables $t_{1}, \ldots, t_{k}$. Exactly as in the case $k=2$ each such integral reduces to $E_{0}\left(M(\varepsilon)^{k-n+1}\right)$ if $n$ of variables are identified, $n=2, \ldots, k$. Thus by the induction hypothesis the error term is $O\left(\varepsilon^{-(k-1) \beta}\right)$. But arguing exactly as in the proof of (4.11) and making use of (4.11) and (4.10) the first term, $J$, on the right-hand side of (4.12) is just

$$
J=k ! \lambda^{k-1} E_{0} \int_{0}^{\infty} h\left(T_{u}^{*}\right) q_{k-1}\left(T_{u}\right) Q(d u, \varepsilon)+k ! O\left(\varepsilon^{-(k-2) \beta}|\log \varepsilon|\right) E_{0} \int_{0}^{\infty} h\left(T_{u}^{*}\right) Q(d u, \varepsilon)
$$

Writing $q_{k-1}\left(T_{u}\right)=q_{k-1}\left(T_{u}^{*}\right)-\left[q_{k-1}\left(T_{u}^{*}\right)-q_{k-1}\left(T_{u}\right)\right]$ and using an argument that is by now familiar we find

$$
J=k ! \lambda^{k} E_{0} \int_{0}^{\infty} h\left(T_{u}^{*}\right) q_{k-1}\left(T_{u}^{*}\right) d u+O\left(\varepsilon^{-(k-1) \beta}|\log \varepsilon|\right) .
$$

Finally combining this with (4.7) and (4.8) we have

$$
E_{0}\left(M(\varepsilon)^{k}\right)=k ! \lambda^{k}[\Gamma(1+\beta k)]^{-1}+O\left(\varepsilon^{-(k-1) \beta}|\log \varepsilon|\right)
$$

and this implies (4.1). Thus Theorem $B$ is, at long last, established.

Note added in proof. The results of this paper and those of [2] are valid for general stable processes of index $\alpha, 1<\alpha \leqq 2$. The proofs in the general case are exactly the same as in the symmetric case once Lemma 3.1 of [2] is established for general stable processes $X$ of index $\alpha$. However, a careful examination of the proof of this lemma in the symmetric case (which goes back to Kac) reveals that exactly the same argument works in the general case. See also a forthcoming paper of C. J. Stone in the Illinois Journal of Mathematics. 


\section{REFERENCES}

1. R. M. Blumenthal and R. K. Getoor, Sample functions of stochastic processes with stationary independent increments, J. Math. Mech. 10 (1961), 493-516.

2. - The dimension of the set of zeros and the graph of a symmetric stable process, Illinois J. Math. 6 (1962), 308-316.

3. K. Ito, On stochastic processes, Japan. J. Math. 18 (1942), 261-301.

4. M. G. Kendall and A. Stuart, The advanced theory of statistics, Vol. I, Hafner, Princeton, N.J., 1958.

5. H. Kesten, Positivity intervals of a stable process, J. Math. Mech. (to appear).

6. M. Loève, Probability theory, 2nd ed., Van Nostrand, 1960.

7. H. Pollard, The completely monotonic character of the Mittag-Leffler function $E_{a}(-x)$, Bull. Amer. Math. Soc. 54 (1948), 1115-1116.

UNIVERSITY OF WASHINGTON, SeATtLE, Washington 